\title{
Aspects éthiques de la prise en charge de personnes démentes
}

\author{
Georg Bossharda, Michelle Salathéb \\ a PD Dr méd., clinique de gériatrie, Hôpital universitaire de Zurich (Président de la sous-commission responsable) \\ b lic. iur., MAE, secrétaire générale adjointe de I'ASSM
}

Les directives de l'ASSM actuellement en vigueur sont en ligne: assm.ch/ Directives

Les anciennes directives peuvent être consultées sous assm.ch $\rightarrow$ Ethique $\rightarrow$ Directives antérieures
Les experts suivants font partie de la sous-commission: PD Dr méd. Georg Bosshard, Zurich (Président, gériatrie) Prof. Driur. Regina Aebi-Müller, Lucerne (droit)

PD Dr méd. Klaus Bally, Bâle (médecine de famille) Dr phil. Stefanie Becker, SSG, Yverdon (psychologie) Dr méd. Daniel Grob, Rheinau (gériatrie) Prof. Dr méd. Christian Kind, Saint-Gall (ancien Président CCE) Prof. Dr rer. medic., Andrea Koppitz, Winterthour (soins de longue durée) Prof. Dr méd. Sophie Pautex, Genève (soins palliatifs)

Dr théol. Heinz Rüegger, MAE, Zurich (éthique) Lic. iur. Michelle Salathé, MAE, Berne, ASSM (droit) Anja Ulrich, MNS, APN, Bâle (soins aigus) Prof. Dr méd. Armin von Gunten, Lausanne (psychiatrie)

\section{Correspondance:} Lic. iur. Michelle Salathé, MAE, secrétaire générale adjointe ASSM

m.salathe[at]samw.ch

La Confédération et les cantons ont fixé, dans la stratégie nationale en matière de démence, des objectifs destinés à améliorer la qualité de vie des personnes atteintes de démence, à réduire les contraintes et à garantir la qualité de la prise en charge. L'ASSM et la Société suisse de gérontologie ont été chargées de la rédaction de directives médico-éthiques concernant la prise en charge et le traitement de personnes atteintes de démence, dans le cadre du projet partiel «Ancrage de direc-

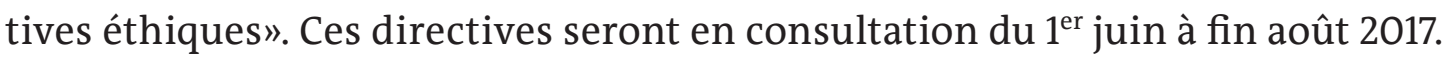

Avec l'allongement de l'espérance de vie, les maladies de démences sont de plus en plus fréquentes. Les questions et les enjeux qui y sont liés sont d'une importance majeure pour le système de santé suisse, mais également pour l'ensemble de notre société. Dès lors, la Confédération et les cantons ont développé, sous l'égide de l'Office fédéral de la santé publique, la Stratégie nationale en matière de démence 2014-2019, qui englobe plusieurs projets partiels concrets. L'ASSM et la Société suisse de gérontologie (SSG) ont été chargées du projet partiel 5.1 "Ancrage de directives éthiques». En février 2015, la Commission Centrale d'Ethique (CCE) de l'ASSM a constitué une sous-commission interprofessionnelle chargée de l'élaboration de ces directives. Son objectif est de proposer une orientation pratique pour la prise en charge et le traitement des personnes atteintes de démence. Dans une prochaine étape, la SSG adaptera et complètera les directives médicoéthiques pour les professions non médicales. Les directives sont articulées autour des problématiques spécifiques auxquelles sont confrontés tous les soignants en charge de personnes atteintes de démence, au-delà du setting (ambulatoire, hôpital, institution de soins) et au-delà des groupes professionnels. Elles s'adressent au médecins, soignants et autres thérapeutes. C'est pourquoi les thèmes s'inscrivant à un niveau supérieur - tels que, par exemple, le développement d'offres de soins de qualité élevée et conformes aux besoins tout au long de la chaîne d'approvisionnement ou le «Skill and Grade mix» des professionnels impliqués - ne sont pas traités dans ces directives. Ces thèmes, qui sont approfondis dans d'autres domaines d'action de la stratégie nationale en matière de démence, sont toutefois des conditions préalables pour que ces directives soient applicables.

Les exigences liées à une prise en charge et un traitement adéquats de personnes atteintes de démence sont élevées. Une démence - souvent combinée à des maladies somatiques et/ou psychiques chroniques (multimorbidité) - est difficilement prévisible et peut s'étendre sur des années. Les directives offrent une aide pratique en abordant les problèmes éthiques à différents niveaux spécifiques. Dès le diagnostic de la maladie, les principes applicables dans les soins palliatifs, entre autres, doivent être pris en considération: qualité de vie primant sur l'allongement de la vie, anticipation, égalité des soins pour tous, échanges interprofessionnels et continuité, communication ouverte et appropriée, soutien lors des processus décisionnels, intégration de l'environnement personnel, multidimensionnalité. La question de l'assistance au suicide n'est abordée qu'en marge de ces directives; ce sujet sera traité en détail dans les directives concernant la prise en charge de personnes en fin de vie, actuellement en révision.

La version des directives «Prise en charge et traitement de personnes atteintes de démence» pour la procédure de consultation a été approuvée par le Sénat en mai 2017. La procédure de consultation publique dure du $1^{\text {er }}$ juin au 31 août 2017. Informations complémentaires: assm.ch/consultation-2017-06 